(Aus der II. medizinischen Abteilung des Wilhelminenspitals in Wien. [Vorstand: Prof. J. Sorgo].)

\title{
Über die Entstehung des saccadierten Atemgeräusches.
}

\author{
Von \\ Dr. Rudolf Peyrer.
}

(Eingegangen an 26. März 1922.)

Seit Jahren schon hat sich das Interesse der medizinischen Forschung von der Erklärung physikalischer Phänomene abgewendet, und heute beherrschen in der internen Diagnostik hauptsächlich chemische Fragen, biochemische und mikrochemische, neben den Problemen der spezifischen Diagnostica das Feld. Der Grund hierfür ist klar : es scheinen eben die früher heißumstrittenen Fragen der Entstehungsweise der Atemgeräusche, der Bedeutung all der auskultatorischen und perkutorischen Erscheinungen so ziemlich gelöst. Doch gibt es auch hier verlassene Stiefkinder, wie das saccadierte Atmen, für das Niemeyer im Jahre 1868 die heute allgemein gültige Erklärung aufstellte: Schwellung der Bronchialschleimhaut, Gewiß ein merkwürdiges Phänomen, das den sonst ununterbrochenen Luftstrom in mehrere Absätze, teilt und das Nachdenken, wie denn das geschehen möge, erregt.

Laënnec, dem Altmeister der Auskultation und Perkussion, war es schon bekannt (Wintrich). Raciborski erklärte es aus einem Krampf der Bronchien und stempelte es bereits zu einem Zeichen beginnender Phthise. Auch dio Franzosen Imbert-Gourbeyre und namentlich Bourgade kultivierten diese Deutung, letzterer besonders meinte, die nächste Erklärung in einer sukzessiven, angestrengten Frweiterung der Lungenzellen finden zu können, wogegen Barth und Roger Adhärenzen der Pleura beschuldigten (Niemeyer). Wintrich (1854) beobachtet es oft als Ausdruck der Ängstlichkeit bei Kindern, alten Leuten; oder bei Krampfzuständen wie im Froststadium verschiedener Fieber oder bei Hyperästhesie der Thoraxwand. Diese Erklärungsversuche faßt Niemeyer (1868) zusammen als Erklärungen des rein beiläufig durch unerhebliche Modifikationen des Respirationsmechanismus entstandenen abgesetzten Atmens, das meiner Meinung nach gar nicht als saccadiertes Atmen bezeichnet werden sollte, sondern etwa als .,stoßweises Atmen", um seine Entstehung durch stoßweises Arbeiten der Atemmuskulatur ins rechte Licht zu setzen, welches Phänomen bei Gesunden wie Kranken in gleicher Weise auftreten kann und für die Lungenpathologie gar kein Interesse hat.

Für das saccadierte Atmen gibt Niemeyer die Erklärung, daß es durch geringen Grad der Anschwellung kleiner Bronchien und Luftzellen und durch Stenosen hervorgerufen werden kann. Auch C. Gerhardt meint, daß bei der Entstehung des saccadierten Atmens ein örtliches Hindernis in den Bronchien den 
Lufteintritt in die Alveolen eines Bezirkes hemmt und zum öfteren während eines Atemzuges überwunden wird. (Außerdem berichtet er 1892 über einen Fall von Pulmonalinsuffizienz mit über der ganzen Lunge hörbarem saccadiertem Atmen und meint, der Pulsus celer im Gebiete der Pulmonalarterie sei die Ursache hievon.) $O$. Vierordt (1889) betont als erster die ,häufig zu findende Pulsrhythmizität": „Es kann ferner in der Umgebung des Herzens und zuweilen bis in die linke Lungenspitze hinauf das Vesikuläratmen der Herzaktion genau entsprechende Absätze zeigen, beruhend auf ungleichem Einströmen der Luft in diese Lungenabschnitte infolge der wechselnden Gestalt des Herzens." Doch gilt ihm dieses ,systolische Vesikuläratmen“ als innerhalb der Norm liegend, während er das saccadierte Atmen als Symptom eines die Bronchien verlegenden Katarrhes deutet. Geigel und Voit (1895) schließen sich ebenfalls Niemeyers Ansicht an (Verengung der Bronchien durch Schwellung oder Sekret). Diese Erklärung findet sich auch bis heute noch in den großen diagnostischen Werken: Brugsch-Schittenhelm (1921) ist der Ansicht, daß „,durch lokale, schnell wieder nachgebende Hindernisse (geringe Schleimhautschwellung, vorübergehende Sekretstauung) der in die Lungenalveolen eintretende Luftstrom auf kurze Zeit unterbrochen wird". Sahli (1920) sucbt den Grund für die ruckweise Hemmung des Atemgeräusches in klappenartigen Schwellungen der Schleimhaut oder ebenfalls in Sekretanhäufungen, die durch den Luftstrom erst zur Seite geschoben werden müssen. Wir wollen später auf diese so simple Theorie der Entstehung saccadierten Atmens durch Schleimhautschwellung und Sekret zurückkommen, denn erst die jetzt zu zitierenden Beobachter versuchen in die einzelnen Eigentümlichkeiten (Persistenz, Lokalisation, Rhythmizität usw.) des Phänomens näher einzugehen und diese genauere Kenntnis ist zur Beurteilung obiger Erklärung nötig.

Volland (1898) betont, daß die einzelnen Abschnitte des saccadierten Atmens stets pulssynchron seien und an diese Feststellung knüpft Otto Hensen (1902) seine Theorie vom Mechanismus des fraglichen Phänomens. Er hatte in der Heilstätte Sonnenburg Gelegenheit, es oft zu beobachten, und zwar fand er es bei $14,5 \%$ seiner tuberkulösen Patienten, jedoch nur bei $2,3 \%$ Gesunder, die auch keine Lungenanamnese darboten. Häufig waren hinten unten Reste pleuritischer Erkrankung wahrnehmbar. Prädilektionsstellen: Links unten hinten, links oben vorn, dann rechts oben vorn, 40 mal links, 25 mal rechts. Meist bestand es lange Zeit hindureh an derselben Stelle. Endlich zitiert er Breckes Arbeit (Die Heilstätte vom R. Kreuz, Grabowsee, Berlin 1899), in der auch festgestellt wird, daß das saccadierte Atmen meist pulsrhythmisch ,nicht nur in der Nähe des Herzens, sondern auch an anderen Teilen des unteren, seltener oberen Brustabschnittes" gefunden wurde. Er bemerkt hierzu: „Auch ich habe den Eindruck bekommen, daß das Vorkommen pulsrhythmisch abgesetzten Atmens bei Tuberkulösen die Regel bildet. In fast allen Fällen, in denen Pulsschlag und Saccade mit genügender Deutlichkeit zutage traten, erschicn mir das Zusammentreffen beider zweifellos." Er unterscheidet im Inspirium meist zwei, drei und vier Saccaden; wenn er durch Laufenlassen des Patienten oder durch Einatmenlassen von Amylnitrit die Herzaktion beschleunigt, so erscheinen entsprechend mehr Saccaden im gleichen Zeitraum. Auf diesen 
Beobachtungen fußend kommt er zu dem zwingenden Schluß, die Saccadierung des Atemgeräusches mit der Herztätigkeit in Verbindung zu bringen, und meint, daß die systolische Erweiterung der Capillaren innerhalb hyperämisch geschwollener Lungenteile den Luftzutritt in die Lungenbläschen in rhythmischen Intervallen hemme und so das saccadierte Atmen bedinge. Abgesehen von den zahllosen Fällen von pulmonaler Hyperämie ohne saccadiertes Atmen - jeder Pneumonie mußte bei Zugrundelegen dieser Ansicht ein, wenn auch noch so kurzes Vorstadium von saccadiertem Atmen aufweisen, bei kardialer Stauung müßte es in der an sich gesunden Lunge zu finden sein sprechen folgende von uns erhobenen Befunde nicht für seine Ansicht:

Fall 1. Der rechte Oberlappen vollständig in ein System alter Kavernen umgewandelt (Obduktionsbefund) und in den unteren Partien des Oberlappens fand man kurz vor dem Exitus noch saceadiertes Atmen.

Fall 2. In der rechten Lunge diffuse relativ spärliche Aussaat von Konglomerattuberkeln mit ausgesprocher Neigung zu fibröser Induration (Obduktionsbefund) saccadiertes Atmen rechts unter der Clavicula.

Fall 3. Links hinten unten saccadiertes Atmen. Obduktionsbefund: Im linken Unterlappen eine frischere brochogene Aussaat: reichlich Konglomerattuberkel in Verkäsung.

Außerdem ist dieser Erklärungsmodus physikalisch unhaltbar, weil in den während der Inspiration sich dehnenden kleinsten Lufträumen die systolische Erweiterung der Capillaren sich im Laufe der Inspiration mit der zunehmenden Erweiterung dieser Räume immer weniger als stenosierendes Hindernis geltend machen müßte, so daß als Folge davon auch die einzelnen Saccaden in ihrer Intensität im Laufe ein und derselben Inspiration einen deutlichen Decrescentocharakter aufweisen müßten, was erfahrungsgemäß nicht der Fall ist.

K. Turban (1899) endlich erklärt die Hemmungen, die das Atmen bei unserem Phänomen erfährt, durch das Zusammentreffen von größeren Partien schlecht oder fast gar nicht atmenden Gewebes einerseits und gesunden Gewebes andererseits, das dann durch ersteres so beeinflußt ist, daß es nur ruckweise sich entfalten kann. Nach Turban ist sein Lieblingssitz wiederum ganz ausdrücklich nicht die Spitze, sondern vorn der 1. und 2. Intercostalraum und hinten die Mitte der Scapula, wobei meist Spitzendämpfung festzustellen ist. Diese Lokalisation ist seiner Meinung nach bedingt durch das ebendort häufige Zusammentreffen eines Infiltrationsbezirkes (Spitzendämpfung) mit normalem Gewebe. Auffallend, schreibt er, sei die Persistenz der Erscheinung, wie gleichmäBig das saccadierte Atmen an den genannten Stellen durch Monate und Jahre bestehen kann, ohne daß jemals irgendwelche Rasselgeräusche auftreten. Abgesehen davon, daß Turban seine Ansicht ganz ohne irgendwelche physikalische Erklärung läßt - und es handelt sich hier doch in erster Linie um physikalische Erscheinungen - , so sprechen die oben zitierten Obduktionsbefunde auch nicht in seinem Sinne. Und endlich ist die nach einwandfreien Beobachtungen auftretende Pulsrhythmizität seiner Meinung als durch sie unerklärt entgegenzuhalten.

Nun können wir noch einmal kurz auf die Erklärungsversuche Niemeyers, Gerhardts, Vierordts, Geigel und Voits, Sahlis und Brugsch-Schittenhelms eingehen. Erstens erklären sie nicht das Phänomen der Pulsrhythmizität. Zweitens spricht der Umstand, daß man das saccasdierte Atmen höchst selten über den Spitzen (Turban), meist aber vorn unter der Clavicula oder hinten in Scapula- 
höhe hört, gegen die Erklärung durch katarrhalische Erscheinungen wie Sekretanhäufungen, Schleimhautschwellungen, denn diese sind am häufigsten über den apicalen Partien zu konstatieren, wo gerade das saccadierte Atmen fehlt. Gegen die Supponierung von Sekretanhäufungen spricht weiter der Umstand der langen Persistenz - während gerade Sekret häufig ausgehustet zu werden pflegt. Auch kann dieses uns höchstens eine Saccade, also: Atemgeräusch Unterbrechung - und wieder Atemgeräusch erklären, wenn es sich zusammenschoppt und dann gewaltsam durchbrochen wird. Eine Erklärung für 3-4 Saccaden, die in genau gleicher Weise und in immer sich gleichbleibendem Rhythmus und Stärke das Atemgeräusch unterbrechen, gibt diese Hypothese nicht. Schleimhautschwellungen hingegen, sofern sie gleichmäßig den Bronchus entlang bestehen, können wohl ïberhaupt nicht zu einer shythmischen Unterbrechung des Atemgeräusches führen, sondern dieses bloß abschwächen, evtl. ganz aufheben, indem sie die verschiedenen Stadien einer Bronchostenose durch Obturation hervorrufen. Klappenartige Schwellungen der Schleimhaut, die nie, auch bei meinen obduzierten Fällen nicht, als Substrat von saccadiertem Atmen vorgefunden wurden, könnten auch nur eine einzige Saccade erklären: ein Einströmen der Luft, die die Klappe spannt, woraus die Unterbrechung des Atemgeräusches resultiert, und ein Durchbrechen der Klappe, ein Umstülpen derselben etwa, so daß nach diesem einmaligen Absetzen die Luft weiterhin ungehindert einströmen kann.

Allerdings kann ich einen Fall berichten, bei dem sich die Annahme einer Sekretanhäufung aufdrängte: Patient H., bei dem neben anderen Zeichen von Lungentuberkulose links hinten unten rauhes Vesiculäratmen gefunden wurde, das einfach abgesetzt war. Von Pulsrhythmus war hier natürlich nicht zu sprechen, nach einem heftigen Hustenstoß war das Phänomen abgeschwächt, am nächsten Tage war es ganz geschwunden.

Diese Erscheinung als saccadiertes Atmen zu bezeichnen liegt uriserer Ansicht nach kein Grund vor. Wir würden den Ausdruck ,einfach abgesetztes Atmen" dafür vorschlagen. Dies könnte dann als Zeichen eines Katarrhes aufgefaßt werden. Den Ausdruck, ,saccadiertes Atmen" jedoch wollen wir für das ungemein eindrucksvolle Phänomen des der Zeitdauer nach rhythmisch, der Stärke der einzelnen Saccaden nach gleichmäßig, und dem Rhythmus nach pulssynchron unterbrochenen Atemgeräusches reserviert wissen. Die Beobachtung der Pulsrhythmizität, die schon manche Beobachter vor uns betonten, die mein verehrter Chef, Herr Prof. Sorgo, konstant fand, und die auch mir immer wieder aufs neue imponierte, und daneben die ebenso häufig schon betonte Persistenz der Erscheinung lie $B$ uns mit zwingender Notwendigkeit an ein pathologisch-anatomisch womöglich feststellbares Substrat denken, das irgendwie in einer besonders innigen Verbindung zwischen Herz und Lunge liegen mußte. Vorerst durchforschte ich die Röntgenbefunde der von mir beobachteten Fälle von saccadiertem Atmen und fand häufig einen Hinweis auf perikardial-pulmonale Adhäsionen, und als endlich drei von mir beobachtete Fälle mit saccadiertem Atmen zur Obduktion kamen und bei jedem dieser Fälle eine Accretio cordis auf der Seite gefunden wurde, die dem Auftreten des saccadierten Atmens entsprach, war die Idee nahegelegt, daß das echte, puls- 
rhythmische, lange Zeit persistierende saccadierte Atemgeräusch ein Symptom einer flächenhaften Accretio cordis mit der Pleura pulmonalis ist. Einige Krankengeschichten mögen diese Mitteilung erhärten:

1. Pat. A. K., aufgenommen am 22. XI. 1921. Stark abgemagert, leicht eyanotisch; die rechte Seite zeigte von der Basis nach aufwärts Schallverkürzung, die am 5 . Dorn in Dämpfung überging, darüber trockene bronchitische Geräusche und daneben klingendes Rasseln. Vorn Dämpfung bis zur 2. Rippe mit hauchendem Exspirium. Unterhalb, also in den unteren Partien des rechten Oberlappens deutlich pulsrhythmisch saccadiertes Atmen, das jedoch auf den Mittellapen nicht übergriff. Links nur die Spitze gedämpft, klingendes Rasseln. Der 1. Unterlappen emphysematös gebläht, das Herz fast gänzlich überlagernd, rauhes Ves.-Atmen, aber trotz des innigen Kontaktes keine Spur einer Saccadierung des Atemgeräusches. Am 26. I. waren Ödeme aufgetreten, die Herztätigkeit sichtlich erlahmt und auch das sacc. Atmen nicht so deutlich. Am 1. XII. die Herztöne kräftig, die Ödeme zurückgegangen und das sacc. Atmen sehr deutlich hörbar. Der Obduktionsbefund vom 5. XII. (Dr. Österlin) ergab neben all den übrigen schweren Veränderungen eine Accretio cordis rechts am Oberlappen von flächenhaftem Charakter, gegen die Basis zu strangförmig.

2. Pat. A. P., aufgenommen am 2. X. 1921. Hochgradig abgemagert zeigte er rechts hinten vom 9. Dorn, links vom 7. Dorn aufwärts Dämpfung mit feuchtem Rasseln. Rechts vorn oben supra- und infraclavieulare Dämpfung mit Wintrichschen Schallwechsel und amphorischem Atmen. Über dem normal begrenzten Herzen an der Aorta ein oberflächliches, schabendes, leises systolisches und lauteres diastolisches Geräusch, das unbedingt accidentellen Charakter trug. Bei einer späteren Untersuchung konstatierten wir rechts vorn zwischen der 2. und 4. Rippe sacc. Atmen. Der Röntgenbefund vom 5. X. (Dr. Fleischner) ergab unter anderem eine pleuropericarditische Schwarte auf der rechten Seite und durch die Autopsie am 11. I. 1922 (Dr. Österlin) wurde eine partielle flächenhafte Accretio cordis rechts gefunden, die uns nun analog dem ersten Fall das sacc. Atmen aber auch das wohl durch den gleichen Mechanismus entstandene Aortengeräusch erklärte. Das Herz zeigte keine Klappenveränderung.

3. Pat. R. Ch. zeigte bereits bei der Aufnahme (23. VI. 1921) einen ausgedehnten Zerfallsprozeß der linken Lunge. Vorn Dämpfung, subclaviculär Kavernensymptome; rückwärts ebenfalls Dämpfung bis zum Angulus mit klingendem Rasseln, unterhalb der Spina saccadiertes bronchovesiculäres Atmen, die untere Grenze unverschieblich. Rechts wenige nicht klingende Rasselgeräusche über der schallverkürzten Spitze. Der Röntgenbefund (Dr. Fleischner) vom 27. VI. erwähnt: „Das linke Zwerchfell ist zeltförmig hochgezogen, steht fast still. Das Herz (bei der Perkussion nicht abgrenzbar) weit links verlagert." "Ein Pneumothoraxversuch gibt dem Röntgenologen Gelegenheit, die Verwachsungsverhältnisse noch genauer zu beobachten: Links unten vorn, z. T. axillar eine auffallend helle, gut handtellergroße Stelle durch zarten Saum begrenzt, bei Schrägstellung deutlich als abgesackte, vorn z. T. vor dem linken Herzen liegende Luftkammer erkennbar. Sin. phren, cost. frei. Das 1. Zwerchfell hoch, fast unbeweglich, angedeutet paradoxe Atmung (Dr. Fleischner am 6. VII.). Pat. wird auf eigenen Wunsch entlassen. Er kommt wieder am 24. XI. 1921, verfallen und hoch fiebernd. Nun ist das Atemgeräusch links hinten so abgeschwächt, daß es nicht zu differenzieren ist. Die Autopsie (Dr. Schuberth) am 6. XII. 1921 ergibt neben dichten Verwachsungen beider Lungen mit der Brustwand eine Accretio cordis beiderseits und mediastinitische Verwachsungen. - Daß das sacc. Atmen nur links hinten wahrnehmbar war, erkläre ich mir durch die sehon in den oben angeführten Röntgenbefunden festgestellten Verwachsungen, die das Herz nach links hinten verzogen. (Vorn war freier Raum für Pneumothorax), weshalb dieses seine Lokomotionsbestrebungen gerade in dieser Richtung geltend machen mußte, aus der es in seiner Stellung festgehalten wurde.

4. Ein weiterer Fall, Pat. J. Z., weist ebenfalls röntgenologisch mediastinale Schwarten links rückwärts auf (Befund vom 10. VIII. 1921, Dr. Fleischner): Im Herzschatten ein flächiger, mäßig dichter Schatten, etwa drei Querfinger von der Mittellinie scharf begrenzt, nicht pulsierend, hinter dem Herzen liegend: Schwarte nach Pleuritis mediastin, post. sin. Er suchte am 6. VI. 1920 die Abtcilung zum ersten Male auf und schon damals wurde sace. Atmen über der ganzen l. Lunge, am stärksten links hinten unten vernommen, das in der 
Krankengeschichte am 26. VII., 8. IX., 14. XI. immer wieder ausdrücklich vermerkt ist. Dann verließ er das Spital. Zum zweiten Male am 8. VIII. 1921 aufgenommen war das sacc. Atmen - in gleicher Weise links hinten unten und angedeutet auch links vorn zu finden. Beim Herzbefund ist angemerkt ein systol. Geräusch über den Gefäßen, besonders über der Pulmonalis.

5. Pat. J. R. endlich zeigt sacc. Atmen rechts unten vorn, korrespondierend dazu der Röntgenbefund (Dr. Fleischner): Im rechten Herzleberwinkel weit vorn in Sinus ein erbsengroßer kalkdichter Herdschatten (Primärherd?). Dieser Winkel flächig mäßig dicht verschattet, Stränge vom Vorhofskontur zum Zwerchfell; der mediale 'Teil des Zwerchfells bleibt bei der Atmung zurück: Pleuropericard. Schwielen rechts.

6. Pat. E. K. zeigte linkerseits weiter vorgeschrittene Veränderungen der oberen zwei Drittel des Oberlappens und der Unterlappenspitze, in der linken Axilla sacc. Atmen. Die Röntgenuntersuchung (Dr. Fleischner) hatte "links am Herzkontur Zeltbildung (Accretio)" ergeben und eine eingeholte orthodiagraphische Skizze zeigt bei etwas aus der dorsoventralen in die erste Schrägstellung gedrehte Haltung eine kleine Zacke mit daran ansetzendem etwa $2 \mathrm{~cm}$ lateral nach außen verlaufendem Strang am linken Herzrand in der Höhe der dritten Rippe vorn, also lokal in Übereinstimmung mit der in der Axilla festgestellten Zone sacc. Atmens.

7. Pat. J. V. zeigte beiderseitige Spitzenprozesse, außerdem rechts vorne von der 2. bis 5. Rippe neben dem Sternum sacc. Atmen. Über dem Herzen an der Spitze ein lautes schabendes systolisches Geräusch, die Grenzen normal, kein accent. 2. Pulm. Ton. Der Röntgenbefund (Dr. Fleischner) ergab: An beiden Zwerchfellen Zeltbildung. Der rechte Herzleberwinkel durch einen pulsierenden Schatten gedeckt (kleiner Erguß ?). Herz klein.

8. Pat. J. W. zeigte links hinten unten verschärftes Inspirium, verlängertes Exspirium; seitlich und vorn links, ebenso vorn rechts sacc. Atmen. Der Röntgenbefund ergab: Pleuropulmonaler ProzeB im linken Unterlappen mit Kavernen. Zeltbildung neben dem Herzen; und die orthodiagraphische Skizze zeigte beiderseits ausgefüllten Herzzwerchfellwinkel, also pleuropericardiale Verwachsungen.

Bemerkt sei zu den Fällen, bei denen kein Obduktionsbefund, wohl aber ein Röntgenbefund vorliegt, daß es sehr schwer, ja in vielen Fällen unmöglich ist, eine flächenhafte Accretio cordis röntgenologisch nachzuweisen. . Doch sieht man mitunter bei ganz genauem Eingehen während der Herzaktion kleine Zacken vom Herzkontur in die Lunge hineinziehen, die evtl. in einer gewissen Körperstellung erst deutlich werden. Zuweilen freilich fällt schon bei der ersten Betrachtung eine Zacke ins Auge, die strangförmig ins Lungengewebe hineinzieht und durch etwa mitgeteilte Pulsation ihre Zusammengehörigkeit mit dem Herzbeutel beweisen kann, der Ausdruck einer vorangegangenen Schädigung, die nicht nur Perikard und Pleura, sondern auch die Lunge betraf. Ein weiteres Zeichen, das eine Pleuropericarditis adhaesiva anzunehmen gestattet, ist die strangförmige oder flächige Ausfüllung der Herzzwerchfellwinkel, die man auch während der Pulsation deutlich sieht. So kann man mit dem Röntgenapparate und zwar bei der Durchleuchtung viel eher als bei Aufnahmen pleuroperikardiale Adhäsionen oftmals mit Sicherheit diagnostizieren.

Nachdem nun der Zusammenhang von saccadiertem Atmen und Pleuropericarditis adhaesiva an mehreren Fällen nachgewiesen wurde, will ich auch die Grundlagen zum mechanischen Verstehen dieser Kombination skizzieren:

Die Veränderungen der Herzgröße und die Herzbewegungen während der rhythmischen Folge von Systole und Diastole wurden genauer von Bamberger, Köllicker, F. Ernst und Riegel neben anderen Forschern studiert und deren Beobachtungen teils an Kaninchen, teils an Menschen entnehmen wir, daß das Herz während der Systole sich verkleinert, indem der transversale Durch- 
messer sich verschmälert und die Basis sich der Spitze nähert, während nur der Durchmesser von vorn nach hinten zunimmt. Dieser Volumsänderung parallel geht eine Lokomotion, indem sich das ganze Herz während der Systole längs der inneren Thoraxwand von rechts oben nach links und abwärts bewegt, so daß jeder einzelne Punkt der Herzoberfläche bei der Systole tiefer rückt. Da gleichzeitig Basis und Spitze sich nähern, macht die Basis infolge der Verkürzung des Längsdurchmessers viel größere Exkursionen. Im Gegensatz zum Herzen, das Eigenbewegung besitzt, muß die Lunge dem ihr dargebotenen Raume sich anpassen und erreicht dies unter normalen Verhältnissen, wenn keine Verwachsungen bestehen, in idealem Maße so, daß vermöge ihrer Elastizitä.t und des freien Gleitens ihrer Oberfläche am Herzen entlang die Bewegungen des Herzens von der ganzen Lunge aufgefangen werden, so daß der auskultatorische Effekt gleich Null sein muß und nur geringe Bewegungen der Luftsäule in den zuführenden Atemwegen anläßlich der Herzaktion mittels subtiler Apparate konstatiert und gemessen werden können (Klewitz und Baumann), wie ja auch jede Inspirationsbewegung des Brustkorbes nicht nur die Randpartien der Lunge, sondern diese allseitig ausdehnt. Ist jedoch die innigere Anheftung der Lunge an das Herz in Gestalt einer Pleuropericarditis adhaesiva gegeben, so wird das Perikard, das dem Herzen folgt, auch die verwachsenen Lungenpartien elektiv mit sich nehmen und ihr, und eben gerade nur ihr Spannungsverhältnis in eingreifender Weise ändern, so daß durch eine derartige Utbertragung der kardialen Bewegungen ein rhythmisches Ausdehnen und Zusammenziehen der innig angehefteten Lungenteile erfolgt, die ebenso pulsrythmisch das Atemgeräusch beeinflussen müssen. Durch diesen Mechanismus wird die Persistenz und die Rhythmizität des saccadierten Atmens ebenso erklärt wie seine Lieblingslokalisation in der Höhe der Herzbasis und gleichzeitig ein Licht auf den Entstehungsmechanismus akzidenteller Herzgeräusche geworfen, die ja auch meistens über der Herzbasis gefunden werden und, wie zwei meiner Patienten zeigen, mit saccadiertem Atmen kombiniert sein können. Ein dritter hierher gehörender Patient J.Z., der ebenfalls saccadiertes Atmen vorn beiderseifs neben der Herzbasis zeigt, weist über der Aorta ein deutlich schabendes Geräusch in der Diastole auf, das bei leisem Atmen sehr verstärkt über Aorta, Pulmonalis und Tricuspidalis zu hören ist, im Liegen beinahe verschwindet. Kein Pulsus celer, kein Capillarpuls usw. Der Röntgenbefund bringt in diesem Falle keinen Aufschluß wegen allzu dichter Hiluszeichnung und Herdschatten. Der rechte Herzleberwinkel ist von Verdichtungen ausgefült, es sind also auch in diesem Falle Accretionen zu vermuten. So eröffnet sich die Frage, ob nicht mindestens ein Teil der akzidentellen Herzgeräusche durch eine Pleuropericarditis adhaesiva bedingt ist, welcher Frage wir weiterhin genaue Aufmerksamkeit widnen werden. Erwähnt sei, daß schon Friedreich 1861 in seiner Arbeit über Herzkrankheiten den ,systolischen und diastolischen Lungengeräuschen" (kardiopulmonale Ger. Potains) eingehendere Aufmerksamkeit widmet und meint, daß durch bestehende Verwachsungen und Fixationen einzelner Lungenteile mit der äußeren Fläche des Pericards sehr wohl derartige Geräusche bedingt sein könnten. Dieser Erklärungsmodus wurde wohl von den späteren Beobachtern .kardiopulmonaler Geräusche nicht aufgegriffen, doch glaube ich ernstlich auf ihn hinweisen zu müssen. 
Wichtig ist weiter die Kombination von saccadiertem Atmen und Schmerzen, die zuweilen von Patienten in den betreffenden Abschnitten der Lungenherzränder angegeben werden und auf Grund unserer Anschauung unschwer als durch Verwachsungen bedingt Erklärung finden.

\section{Zusammenfassung.}

Die bisher bekannten Erklärungsmodi des saccadierten Atemgeräusches entsprechen den gefundenen Tatsachen nicht. Persistenz, Lokalisation und Pulsrhythmizität weisen auf pathologisch-anatomisch feststellbare Verbindungen zwischen dem Herzen und den in Frage kommenden Lungenabschnitten. Solche pleuroperikarditische Adhäsionen konnten vom Verfasser in 3 Füllen mit saccadiertem Atmen durch Obduktion festgestellt werden, in weiteren Fällen wurden sie röntgenologisch beobachtet, so daß die Entstehung des saccadierten Atmens durch Accretio cordis bewiesen erscheint. Weiters weist Verfasser auf die in einigen Fällen gleichzeitig beobachteten akzidentellen Herzgeräusche hin und möchte auch der Entstehung mindestens eines Teiles der kardicpulmonalen Geräusche eine Accretio cordis zugrunde legen.

\section{Literaturverzeichnis.}

Wintrich, Krankheiten der Respirationsorgane. Virchow, Handb. 5, Abt. I. Erlangen 1854. - N. Friedreich, Krankheiten des Herzens. Virchow, Handb. 5, Abt. II, Erlangen 1861. $P$. Niemeyer, Handbuch der theoretischen und praktischen Perkussion und Auskultation. Erlangen 1868. - P. Niemeyer, Grundriß der Perkussion und Auskultation. Erlangen 1871. - C. Gerhardt, Lehrbuch der Auskultation und Perkussion. Tübingen 1876. - C. Gerhardt, dasselbe, 5. Aufl. Tübingen 1890. - C. Gerhardt, Über SchluBunfähigkeit der Lungenarterienklappen. Verh. d. 11. Kongr. f. inn. Med. 1892, S. 296. - $O$. Vierordt, Diagnostik der inneren Krankheiten. Leipzig 1889. - Geigel und Voit, Lehrbuch der klin. Untersuchungsmethoden. Stuttgart 1895. - Volland, Die Lungenschwindsucht. Tübingen 1898, zit. nach Hensen. K. Turban, Beiträge zur Kenntnis der Lungentuberkulose. Wiesbaden 1899. - Otto Hensen, Über sacc. Atmen. Dtsch. Arch. f. klin. Med. 1902, S. $230 \mathrm{ff}$. - Wenckebach, Über pathologische Beziehungen zwischen Atmung und Kreislauf. Volkmanns Samml. klin. Vorträge 465/6. 1907. - Striimpell, Lehrbuch der speziellen Pathologie und Therapie innerer Krankheiten. 19. Aufl. 1904, I. Bd. - Sahli, Klinische Untersuchungsmethoden. 1, 6. Aufl. LeipzigWien 1920. - Brugsch-Schittenhelm, Klinische Untersuchungsmethoden 1921. - Bamberger, Beiträge zur Physiologie und Pathologie des Herzens. Virchows Arch. f. pathol. Anat. u. Physiol. 9. 1856. - Bamberger, Lehrbuch der Krankheiten des Herzens. Wien 1857. F. Ernst, Studien über die Herztätigkeit usw. Virchows Arch. f. pathol. Anat. u. Physiol. 9. 1856. - L. Brauer, Untersuchungen am Herzen. Verh. d. Kongr. f. inn. Med. 1904, S. 187. Skoda, Über unerklärliche Herzgerüusche. Allg. W. med. Zeitschr. 1863, Nr. 33. - H. Müller, Ǔber cardiopulmonale Geräusche. Volkmanns Samml. klin. Vortr., Neue Folge 500/501, 1908. - Romberg, Lehrbuch der Krankheiten des Herzens und der Blutgefäße, 2. Aufl. Stuttgart 1909. - Lang, Bewegung der Brustwand und des Epigastriums. Dtsch. Arch. f. klin. Med. 108. - v. Hoffmann, Dissertation über die klinischen Symptome der adhäs. Perikarditis. Berlin 1905. - Riegel, Die Diagnose der Pleuraverwachsungen. Volkm. Samml. klin. Vortr. 177. - Landois, Lehrbuch der Physiologie des Menschen, und Tagebl. d. Naturf.Vers. Hamburg 1876. - J. Schütze, Röntgenbeobachtungen bei extraperik. Verwachsungen. Berl. klin. Wochenschr. 1921, Nr. 36. - Flatauer, Beeinträchtiungg der Herztätigkeit durch perik. Adhäsionen. Ebenda. - v. Achelis, Über adhäsive Perikarditis. Dtsch. Arch. f. klin. Med. I15. 1914. - Riegel, Die Diagnose der Perikardialrerwachsungen. Volkm. klin. Vortr. 177. - Kirch, Zur Klinik der Concretio- und Accretio cordis. Wien. Arch. f. inn. Med. 1920. - Rosenbach, Die Erkrankungen des Brustfells. Nothnagel, Spez. Pathol, u. Therapie, Wien 1899. 